\title{
Generalized energy inequalities and higher multifractal moments
}

\author{
Kenneth Falconer \\ Mathematical Institute, University of St Andrews, North Haugh, St Andrews, \\ Fife, KY16 9SS, Scotland
}

May 22, 2013

\begin{abstract}
We present a class of generalized energy inequalities and indicate their use in investigating higher multifractal moments, in particular $L^{q}$-dimensions of images of measures under Brownian processes, $L^{q}$-dimensions of almost self-affine measures, and moments of random cascade measures.
\end{abstract}

\section{Introduction}

Calculations in fractal geometry often fall into two parts: a geometric part and an analytic part. The geometric part may involve expressing geometric or metric aspects of a problem in mathematical terms leading to an analytic argument to estimate the integrals, sums, etc. so obtained. There are a range of analytic methods that are applicable to problems in fractal geometry of apparently different natures, for example covering or potential theoretic methods for estimating dimensions. We will look at an analytic technique which extends the potential theoretic method to higher moments and give several applications.

\section{$2 \quad L^{q}$-dimensions and images of measures}

Coarse multifractal analysis reflects the asymptotic behavior of the moment sums of measures over small grid cubes. Let $\mathcal{M}_{r}$ be the set of mesh cubes of side $r$, that is cubes in $\mathbb{R}^{n}$ of the form $\left[j_{1} r,\left(j_{1}+1\right) r\right) \times \cdots \times\left[j_{n} r,\left(j_{n}+1\right) r\right)$ where $j_{1}, \ldots, j_{n} \in \mathbb{Z}$. Let $\mu$ be a Borel measure of bounded support on $\mathbb{R}^{n}$. Define the $q$-th power moment sum of $\mu$ by

$$
M_{r}(q)=\sum_{C \in \mathcal{M}_{r}} \mu(C)^{q}
$$

momentdef

The $L^{q}$-dimension or generalized $q$ dimension of $\mu$ is given by

$$
D_{q}(\mu)=\frac{1}{q-1} \lim _{r \searrow 0} \frac{\log M_{r}(q)}{\log r} \quad(q>0) .
$$


If this limit does not exist we may still take lower or upper limits to get the lower and upper $L^{q}$-dimensions:

$$
\underline{D}_{q}(\mu)=\frac{1}{q-1} \varliminf_{r \searrow 0} \frac{\log M_{r}(q)}{\log r} \quad \text { and } \quad \bar{D}_{q}(\mu)=\frac{1}{q-1} \varlimsup_{r \searrow 0} \frac{\log M_{r}(q)}{\log r} .
$$

The definitions $\left(\frac{1}{2.2}\right)$ and $(2.3)$ are unchanged ifwe replace the moment sum by a moment integral

$$
M_{r}(q)=\int \mu(B(x, r))^{q-1} d \mu(x) \quad(q>0),
$$

momentint

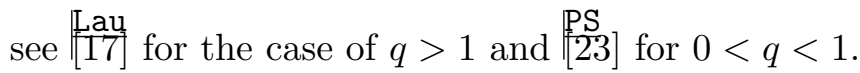

Often of interest are the dimensions of the image of a set or the generalized dimensions of the image of a measure under a parameterized family of mappings. Let $X$ be a metric space, and let $x_{\omega}: X \rightarrow \mathbb{R}^{n}$ be a family of continuous mappings where $\omega \in \Omega$ for some parameter space $\Omega$. Let $\mu$ be a Borel measure on $X$ and let $\mu_{\omega}$ be its image measure under $x_{\omega}$, so

$$
\mu_{\omega}(A)=\mu\left(x_{\omega}^{-1}(A)\right) \quad\left(A \in \mathbb{R}^{n}\right)
$$

or

$$
\int f(x) d \mu_{\omega}(x)=\int f\left(x_{\omega}(t)\right) d \mu(t) \quad\left(f: \mathbb{R}^{n} \rightarrow \mathbb{R}\right) .
$$

For a basic example, $x_{\omega}$ might be orthogonal projection from $\mathbb{R}^{m}$ onto a line $L_{\omega}$ (which we may identify with $\mathbb{R}$ ) in direction $\omega$, with $\mu_{\omega}$ the corresponding projection of the measure $\mu$ on $\mathbb{R}^{m}$ onto $L_{\omega}$.

Now suppose $(\Omega, P, \mathcal{F})$ is a probability space and write $E$ for expectation. One way of obtaining lower estimates for $L^{q}$-dimensions of $\mu_{\omega}$ valid for almost all $\omega$ is to bound the mean moment integrals. When $q \geq 2$ is an integer:

$$
\begin{aligned}
& \mathrm{E} \int \mu_{\omega}(B(x, r))^{q-1} d \mu_{\omega}(x) \\
& =\mathrm{E} \int \mu_{\omega}\left\{y_{1}:\left|x-y_{1}\right| \leq r\right\} \ldots \mu_{\omega}\left\{y_{q-1}:\left|x-y_{q-1}\right| \leq r\right\} d \mu_{\omega}(x) \\
& =\mathrm{E} \int \mu\left\{t_{1}:\left|x_{\omega}(t)-x_{\omega}\left(t_{1}\right)\right| \leq r\right\} \ldots \mu\left\{t_{q-1}:\left|x_{\omega}(t)-x_{\omega}\left(t_{q-1}\right)\right| \leq r\right\} d \mu(t) \\
& =\mathrm{E} \int \ldots \int \chi_{\left\{\left|x_{\omega}(t)-x_{\omega}\left(t_{j}\right)\right| \leq r \text { for all } j\right\}}\left(t_{1}, \ldots, t_{q-1}, t\right) d \mu\left(t_{1}\right) \ldots d \mu\left(t_{q-1}\right) d \mu(t) \\
& =\int \ldots \int \mathrm{P}\left\{\left|x_{\omega}(t)-x_{\omega}\left(t_{j}\right)\right| \leq r \text { for all } j\right\} d \mu\left(t_{1}\right) \ldots d \mu\left(t_{q-1}\right) d \mu(t) .
\end{aligned}
$$

We may be able to use the geometry of the situation to estimate $\mathrm{P}\left\{\left|x_{\omega}(t)-x_{\omega}\left(t_{j}\right)\right| \leq\right.$ $r$ for all $j$ \}, which depends on the relative closeness of the $t_{1}, \ldots, t_{q-1}, t$ in the metric space. For example, with $x_{\omega}: \mathbb{R}^{m} \rightarrow L_{\omega}$ as projection onto the line $L_{\omega}$ where $\omega \in \Omega$ is distributed according to the natural invariant measure on the space of directions $\Omega$, the probability $\mathrm{P}\left\{\left|x_{\omega}(t)-x_{\omega}\left(t_{j}\right)\right| \leq r\right.$ for all $\left.j\right\}$ is affected more, but not exclusively, by the $t_{j}$ that are furthest from $t$, see Figure 1 . In particular, bounding $(2.5)$ by const. $r^{s(q-1)}$ may lead to a lower bound of $s$ for the $L^{q}$-dimension of $\mu_{\omega}$ for almost all $\omega$.

In the case when $q=2$ the integral (2.5) may be estimated by

$$
\iint \mathrm{P}\left\{\left|x_{\omega}(t)-x_{\omega}\left(t_{1}\right)\right| \leq r\right\} d \mu\left(t_{1}\right) d \mu(t) \leq \iint \mathrm{E}\left(\frac{r^{s}}{\left|x_{\omega}(t)-x_{\omega}\left(t_{1}\right)\right|^{s}}\right) d \mu\left(t_{1}\right) d \mu(t)
$$




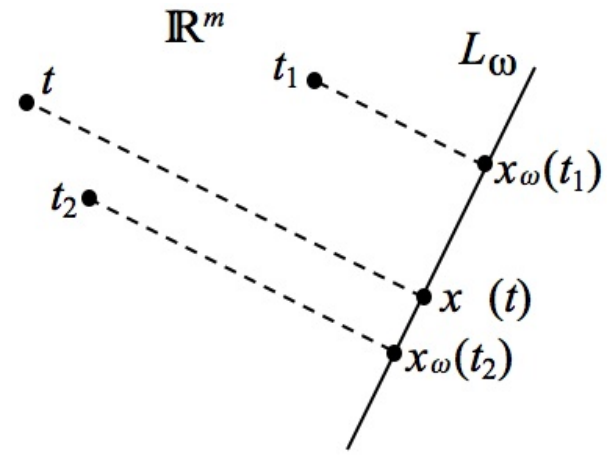

Figure 1: Projection of three points onto a line parameterized by $\omega$

for all $s>0$. This expectation can often be estimated using a transversality argument which results in an energy-type integral. The classic case of this is in the projection theorems, see for example $[7,21]$ for the projection case and $[24]$ for a more general setting.

\section{The main inequality}

maíméneQ

In this section we consider an approach to estimating integrals such as (2.5) for $q>1$ and present an inequality which we may be applied in various settings. It is convenient to take $X$ to be the symbolic space on a set of $m \geq 2$ symbols, $\Lambda \equiv\{1, \ldots, m\}$. Thus $\Lambda^{k}$ consists of the words of length $k$ for $k \geq 0$ and we write $\Lambda^{*} \equiv \cup_{i=0}^{k} \Lambda^{k}$ which we identify with the vertices of the $m$-ary rooted tree in the usual way. The infinite sequences, identified with the boundary of the tree, are denoted by $\Lambda^{\infty}$. For $\mathbf{i}=i_{1}, \ldots, i_{k} \in \Lambda^{*}$ we write $\mathbf{i}=k$ for the length of the word $\mathbf{i}$. For $\mathbf{i} \in \Lambda^{*}$ and $\mathbf{j} \in \Lambda^{*} \cup \Lambda^{\infty}$ we write $\mathbf{j} \succ \mathbf{i}$ to mean that $\mathbf{i}$ is an initial segment of $\mathbf{j}$. The cylinders are the sets $C_{\mathbf{i}}=\left\{\mathbf{j} \in \Lambda^{\infty}: \mathbf{j} \succ \mathbf{i}\right\}$ for each $\mathbf{i} \in \Lambda^{*}$. The cylinders provide a basis for the natural topology on $\Lambda^{\infty}$.

Write $\mathbf{j}_{1} \wedge \mathbf{j}_{2} \in \Lambda^{*}$ for the join of $\mathbf{j}_{1}, \mathbf{j}_{2} \in \Lambda^{\infty}$, that is the longest $\mathbf{i} \in \Lambda^{*}$ such that $\mathbf{j}_{1} \succ \mathbf{i}$ and $\mathbf{j}_{2} \succ \mathbf{i}$. For an integer $q \geq 2$ we define the set of join points $\mathbf{i}_{1}, \ldots, \mathbf{i}_{q-1} \in \Lambda^{*}$ of $\mathbf{j}_{1}, \mathbf{j}_{2}, \ldots, \mathbf{j}_{q} \in \Lambda^{\infty}$ to be the set

$$
J\left(\mathbf{j}_{1}, \mathbf{j}_{2}, \ldots, \mathbf{j}_{q}\right)=\left\{\mathbf{j}_{i} \wedge \mathbf{j}_{j}: 1 \leq i<j \leq m\right\},
$$

see Figure 2 . This set will always consist of exactly $q-1$ points provided that they are counted according to multiplicity, that is if there are $r$ distinct points $\mathbf{j}_{i_{1}}, \ldots, \mathbf{j}_{i_{r}}$ such that $\mathbf{i}=\mathbf{j}_{i_{p}} \wedge \mathbf{j}_{i_{q}}$ for all $1 \leq p<q \leq r$ then $\mathbf{i}$ is counted as a join point with multiplicity $r-1$. (If $m=2$, corresponding to a binary tree, then all join points have multiplicity 1.)

In bounding expressions such as $(2.5)$, where we now take $X=\Lambda^{\infty}$ so that the $t_{i} \in X$ are replaced by $\mathbf{j} \in \Lambda^{\infty}$, a generalised transversality argument may lead to an estimate of the form

$$
\mathrm{P}\left\{\left|x_{\omega}\left(\mathbf{j}_{q}\right)-x_{\omega}\left(\mathbf{j}_{j}\right)\right| \leq r \text { for all } j\right\} \leq F\left(\mathbf{j}_{1}, \mathbf{j}_{2}, \ldots, \mathbf{j}_{q}\right)
$$

where $F$ may be expressed as a product over the join points

$$
F\left(\mathbf{j}_{1}, \mathbf{j}_{2}, \ldots, \mathbf{j}_{q}\right)=f\left(\mathbf{i}_{1}\right) f\left(\mathbf{i}_{2}\right) \ldots f\left(\mathbf{i}_{q-1}\right) \text { where }\left\{\mathbf{i}_{1}, \ldots, \mathbf{i}_{q-1}\right\}=J\left(\mathbf{j}_{1}, \mathbf{j}_{2}, \ldots, \mathbf{j}_{q}\right),
$$




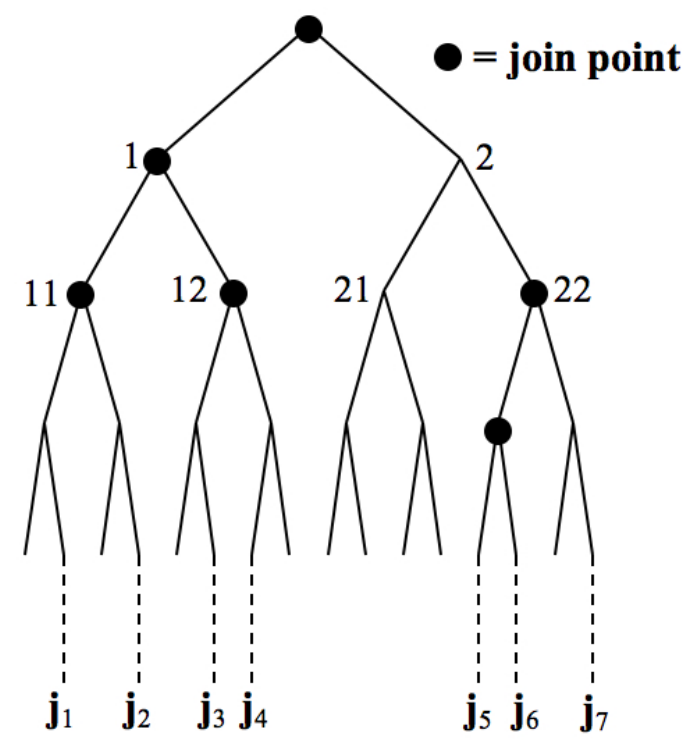

Figure 2: A set of 7 points in $\Lambda^{\infty}$ with their 6 join points in $\Lambda^{*}$

tree

for some $f: \Lambda^{*} \rightarrow \mathbb{R}^{+}$defined on the vertices of the tree. Then (2.5) takes the form

$$
\mathrm{E} \int \mu_{\omega}(B(x, r))^{q-1} d \mu_{\omega}(x) \leq \int \ldots \int F\left(\mathbf{j}_{1}, \mathbf{j}_{2}, \ldots, \mathbf{j}_{q}\right) d \mu\left(\mathbf{j}_{1}\right) \ldots d \mu\left(\mathbf{j}_{q-1}\right) d \mu\left(\mathbf{j}_{q}\right) .
$$

The following theorem estimates this integral in terms of $f$ and the cylinder measures $\mu\left(C_{\mathbf{i}}\right)$.

Theorem 3.1 For each real number $q>1$ there is a polynomial $p$ such that

$$
\int . . \int F\left(\mathbf{j}_{1}, \mathbf{j}_{2}, \ldots, \mathbf{j}_{q}\right) d \mu\left(\mathbf{j}_{1}\right) \ldots d \mu\left(\mathbf{j}_{q}\right) \leq\left(\sum_{k=0}^{\infty} p(k)\left[\sum_{|\mathbf{i}|=k} f(\mathbf{i})^{q-1} \mu\left(C_{\mathbf{i}}\right)^{q}\right]^{\frac{1}{q-1}}\right)^{q-1} .
$$

Proof. We give the proof in the special case when $q=3$, that is

$$
\iiint F\left(\mathbf{j}_{1}, \mathbf{j}_{2}, \mathbf{j}_{3}\right) d \mu\left(\mathbf{j}_{1}\right) d \mu\left(\mathbf{j}_{2}\right) d \mu\left(\mathbf{j}_{3}\right) \leq\left(\sum_{k=0}^{\infty}\left[\sum_{|\mathbf{i}|=k} f(\mathbf{i})^{2} \mu\left(C_{\mathbf{i}}\right)^{3}\right]^{1 / 2}\right)^{2} .
$$

Splitting this integral into a sum over possible pairs of join points, see Figure $\frac{\mid \mathrm{figjoin} 3}{3,}$

$$
\iiint F\left(\mathbf{j}_{1}, \mathbf{j}_{2}, \mathbf{j}_{3}\right) d \mu\left(\mathbf{j}_{1}\right) d \mu\left(\mathbf{j}_{2}\right) d \mu\left(\mathbf{j}_{3}\right) \leq \sum_{\mathbf{i} \in \Lambda^{*}} \sum_{\mathbf{j} \in \Lambda^{*}, \mathbf{j} \succ \mathbf{i}} f(\mathbf{i}) f(\mathbf{j}) \mu\left(C_{\mathbf{i}}\right) \mu\left(C_{\mathbf{j}}\right)^{2} .
$$

firstest

We first estimate the restriction of this double sum over vertices of the tree for given levels $|\mathbf{i}|=k$ and $|\mathbf{j}|=l$ where $0 \leq k<l$; Cauchy's inequality is used at the places 


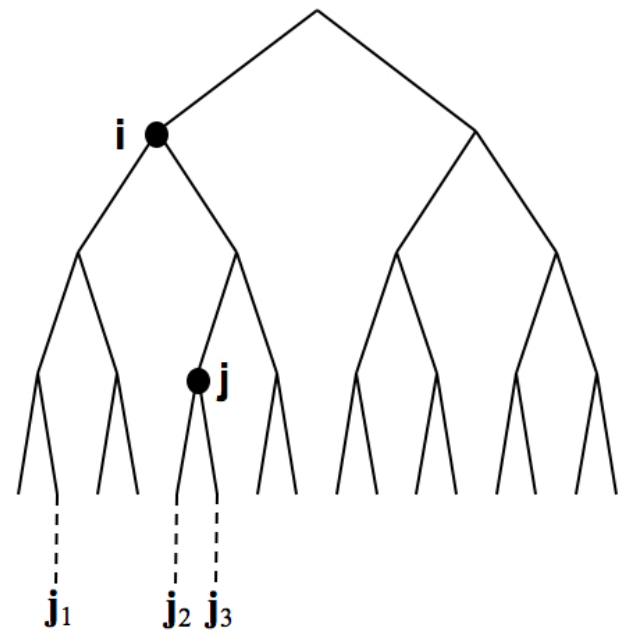

Figure 3: The arrangement of three points in $\Lambda^{\infty}$ the join points used in the proof of Theorem 1

indicated.

$$
\begin{aligned}
& \sum_{|\mathbf{i}|=k} \sum_{|\mathbf{j}|=l, \mathbf{j} \succ \mathbf{i}} f(\mathbf{i}) f(\mathbf{j}) \mu\left(C_{\mathbf{i}}\right) \mu\left(C_{\mathbf{j}}\right)^{2} \\
& =\sum_{|\mathbf{i}|=k}\left[f(\mathbf{i}) \mu\left(C_{\mathbf{i}}\right)\right]\left[\sum_{|\mathbf{j}|=l, \mathbf{j} \succ \mathbf{i}}\left(f(\mathbf{j}) \mu\left(C_{\mathbf{j}}\right)^{3 / 2}\right) \mu\left(C_{\mathbf{j}}\right)^{1 / 2}\right] \\
& \leq \sum_{|\mathbf{i}|=k}\left[f(\mathbf{i}) \mu\left(C_{\mathbf{i}}\right)\right]\left[\left(\sum_{|\mathbf{j}|=l, \mathbf{j} \succ \mathbf{i}} f(\mathbf{j})^{2} \mu\left(C_{\mathbf{j}}\right)^{3}\right)^{1 / 2}\left(\sum_{|\mathbf{j}|=l, \mathbf{j} \succ \mathbf{i}} \mu\left(C_{\mathbf{j}}\right)\right)^{1 / 2}\right] \text { (Cauchy) } \\
& =\sum_{|\mathbf{i}|=k}\left[f(\mathbf{i}) \mu\left(C_{\mathbf{i}}\right)\right]\left[\left(\sum_{|\mathbf{j}|=l, \mathbf{j} \succ \mathbf{i}} f(\mathbf{j})^{2} \mu\left(C_{\mathbf{j}}\right)^{3}\right)^{1 / 2} \mu\left(C_{\mathbf{i}}\right)^{1 / 2}\right] \\
& =\sum_{|\mathbf{i}|=k}\left[f(\mathbf{i}) \mu\left(C_{\mathbf{i}}\right)^{3 / 2}\right]\left[\sum_{|\mathbf{j}|=l, \mathbf{j} \succ \mathbf{i}} f(\mathbf{j})^{2} \mu\left(C_{\mathbf{j}}\right)^{3}\right]^{1 / 2} \\
& \leq\left[\sum_{|\mathbf{i}|=k} f(\mathbf{i})^{2} \mu\left(C_{\mathbf{i}}\right)^{3}\right]^{1 / 2}\left[\sum_{|\mathbf{i}|=k} \sum_{|\mathbf{j}|=l, \mathbf{j} \succ \mathbf{i}} f(\mathbf{j})^{2} \mu\left(C_{\mathbf{j}}\right)^{3}\right]^{1 / 2} \quad \text { (Cauchy) } \\
& =\left[\sum_{|\mathbf{i}|=k} f(\mathbf{i})^{2} \mu\left(C_{\mathbf{i}}\right)^{3}\right]^{1 / 2}\left[\sum_{|\mathbf{j}|=l} f(\mathbf{j})^{2} \mu\left(C_{\mathbf{j}}\right)^{3}\right]^{1 / 2}
\end{aligned}
$$

Summing over all levels $0 \leq k, l$ gives inequality $\left(\begin{array}{l}\text { case } 3.4 \\ 3.4\end{array}\right.$

When $q$ is a larger integer, (3.3eq may be established using an induction on configurations of join points, requiring frequent uses of Hölder's inequality rather than Cauchy's inequality. A further extension of the calculation establishes that $(3.3)$ remains valid for any real number $q>1$, see $[9,11]$ for further details.

In applications $f(\mathbf{i}) \equiv f_{s}(\mathbf{i})$ typically depends on a parameter $s$ such that

$$
\sum_{|\mathbf{i}|=k} f_{s}(\mathbf{i})^{q-1} \mu\left(C_{\mathbf{i}}\right)^{q} \asymp\left(\lambda_{s}\right)^{k}
$$


for some $\lambda_{s}>0$. Combining $\left(\frac{\text { lineq }}{3.3)}\right.$ with $\frac{\text { lexpgendim }}{(3.2) \text { gives }}$

$$
\mathrm{E} \int \mu_{\omega}(B(x, r))^{q-1} d \mu_{\omega}(x) \leq c\left(\sum_{k=0}^{\infty} p(k)\left(\lambda_{s}\right)^{k /(q-1)}\right)^{q-1}
$$

so the value of $s$ for which $\lambda_{s}=1$ is critical for bounding the mean $L^{q}$ dimensions of $\mu_{\omega}$.

\section{Images of measures under Gaussian processes}

$\sec : 3$

For a first application of inequality $\left(\frac{\text { ineq }}{3.3}\right)$, we examine images of measures under certain Gaussian processes. Let $\left\{x_{\omega}:[0,1] \rightarrow \mathbb{R}, \omega \in \Omega\right\}$ be index- $\alpha$ fractional Kah1, ManVN $_{\text {An }}$ Brownian motion $(0<\alpha \leq 2)$ defined on a suitable probability space $\Omega$, see $[2,15$, 20]. It was shown by Kahane $[15]$ that for a Borel set $E \subseteq \mathbb{R}$

$$
\operatorname{dim}_{\mathrm{H}} X(E)=\min \left\{1, \frac{\operatorname{dim}_{\mathrm{H}} E}{\alpha}\right\} \quad \text { a.s., }
$$

where $\operatorname{dim}_{\mathrm{H}}$ denotes Hausdorff dimension. It is natural to seek similar relationships between the generalized dimensions of measures and their images under such processes.

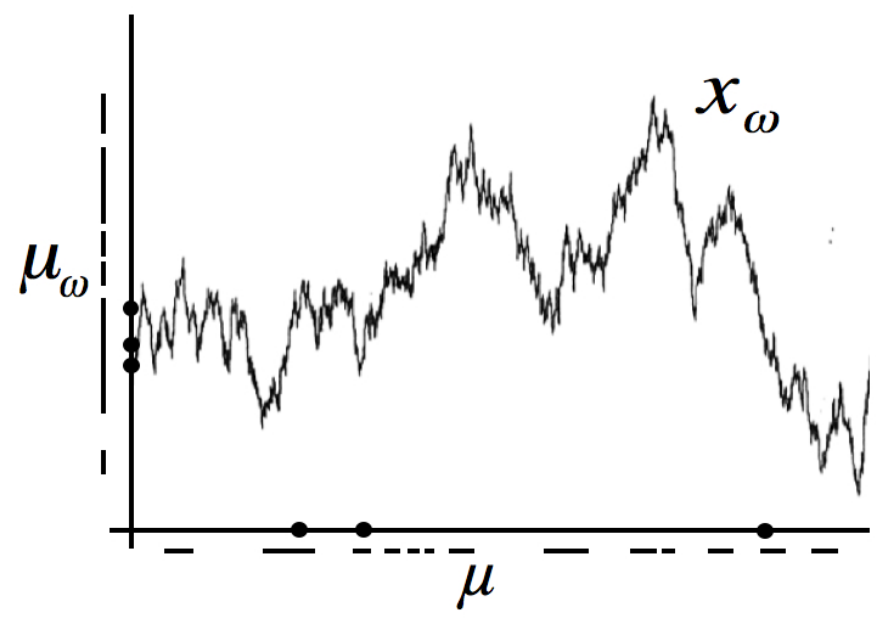

Figure 4: A measure $\mu$ and its image $\mu_{\omega}$ under a process $x_{\omega}$

Theorem 4.1 Let $x_{\omega}:[0,1] \rightarrow \mathbb{R}$ be index- $\alpha$ fractional Brownian motion, let $\mu$ be a finite measure on $[0,1]$ and let $\mu_{\omega}$ be the image of $\mu$ under $x_{\omega}$. Letq $>1$. Assuming that $D_{q}(\mu)$ exists then $D_{q}\left(\mu_{\omega}\right)$ exists almost surely and

$$
D_{q}\left(\mu_{\omega}\right)=\min \left\{1, \frac{D_{q}(\mu)}{\alpha}\right\} \quad \text { a.s.. }
$$

Sketch of proof. Since index- $\alpha$ fractional Brownian motion almost surely satisfies an $(\alpha-\epsilon)$-Hölder condition for all $\epsilon>0$, it follows easily from the definition of $L^{q}$-dimensions that $D_{q}\left(\mu_{\omega}\right) \leq D_{q}(\mu) / \alpha$.

For the opposite inequality we use the local nondeterminism (LND) of fBm. Roughly this states that the variance of $x_{\omega}\left(t_{1}\right)$ conditional on $x_{\omega}\left(t_{2}\right), \ldots, x_{\omega}\left(t_{q}\right)$ is 
comparable with the variance of $x_{\omega}\left(t_{1}\right)-x_{\omega}\left(t_{j}\right)$ for the $j$ for which $\left|t_{1}-t_{j}\right|$ is least), see $[3,25,26]$. It may be shown that the calculations are essentially unaffected if, for a suitably large $m$, we consider the numbers in $[0,1]$ to base $m$ and identify the base $m$ number $0 . a_{1} a_{2} a_{3} \ldots$ with $\left(a_{1}+1, a_{2}+1, a_{3}+1, \ldots\right) \in \Lambda^{\infty}$, so that the hierarchy of $m$-ary subintervals of $[0,1]$ are the cylinders $C_{\mathbf{i}}$ in symbolic space. Using LND inductively we obtain, in symbolic space notation,

$$
\mathrm{P}\left\{\left|x_{\omega}\left(\mathbf{j}_{q}\right)-x_{\omega}\left(\mathbf{j}_{j}\right)\right| \leq r \text { for all } j\right\} \leq c F\left(\mathbf{j}_{1}, \mathbf{j}_{2}, \ldots, \mathbf{j}_{q}\right)
$$

where $F$ is a product over the join points $\mathbf{i}_{1}, \ldots, \mathbf{i}_{q-1} \in J\left(\mathbf{j}_{1}, \mathbf{j}_{2}, \ldots, \mathbf{j}_{q}\right)$ of the form

$$
F\left(\mathbf{j}_{1}, \mathbf{j}_{2}, \ldots, \mathbf{j}_{q}\right)=c r^{s(q-1)} m^{\left|\mathbf{i}_{1}\right| \alpha s} m^{\left|\mathbf{i}_{m}\right| \alpha s} \ldots m^{\left|\mathbf{i}_{q-1}\right| \alpha s}
$$

for any $s>0$, where we have replaced Euclidean distance on $[0,1]$ by the $m$-ary ultrametric $d\left(\mathbf{j}_{1}, \mathbf{j}_{2}\right)=m^{-\left|\mathbf{j}_{1} \wedge \mathbf{j}_{2}\right|}$. In this notation $(2.5)$ becomes

$$
\begin{aligned}
& \mathrm{E} \int \mu_{\omega}(B(x, r))^{q-1} d \mu_{\omega}(x) \\
& \quad \leq c r^{s(q-1)} \int \cdots \int_{\mathbf{i}_{1}, \ldots, \mathbf{i}_{q-1} \in J\left(\mathbf{j}_{1}, \ldots, \mathbf{j}_{q}\right)} m^{\left|\mathbf{i}_{1}\right| \alpha s} m^{\left|\mathbf{i}_{m}\right| \alpha s} \cdots m^{\left|\mathbf{i}_{q-1}\right| \alpha s} d \mu\left(\mathbf{j}_{1}\right) \ldots d \mu\left(\mathbf{j}_{q}\right) .
\end{aligned}
$$

Inequality $\left(\frac{\text { ineq }}{3.3)}\right.$ with $f(\mathbf{i})=f_{s}(\mathbf{i}) \equiv m^{|\mathbf{i}| \alpha s}$ now gives

$$
\mathrm{E} \int \mu_{\omega}(B(x, r))^{q-1} d \mu_{\omega}(x) \leq c r^{s(q-1)}\left(\sum_{k=0}^{\infty} p(k)\left[\sum_{|\mathbf{i}|=k} \lambda_{s, k}\right]^{1 /(q-1)}\right)^{q-1}
$$

where

$$
\lambda_{s, k} \equiv \sum_{|\mathbf{i}|=k} f_{s}(\mathbf{i})^{q-1} \mu\left(C_{\mathbf{i}}\right)^{q}=m^{|\mathbf{i}| \alpha s(q-1)} \sum_{|\mathbf{i}|=k} \mu\left(C_{\mathbf{i}}\right)^{q} .
$$

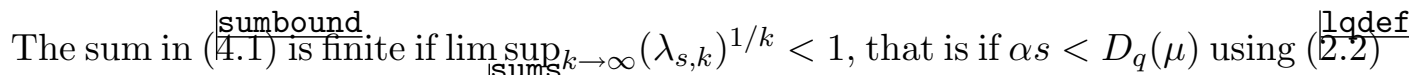
and noting that the sum in $(\overline{4.2})$ is a sum over the $m$-ary mesh intervals of lengths $m^{-|\mathbf{i}|}$ that are identified with the cylinders $C_{\mathbf{i}}$. It follows that if $s_{1}<s<D_{q}(\mu) / \alpha$ then

$$
\begin{aligned}
& \mathrm{E} \sum_{k=1}^{\infty} 2^{-s_{1}(q-1)} \int \mu_{\omega}\left(B\left(x, 2^{-k}\right)\right)^{q-1} d \mu_{\omega}(x) \\
&=\mathrm{E} \int\left(\sum_{k=1}^{\infty} 2^{-s_{1}(q-1)} \mu_{\omega}\left(B\left(x, 2^{-k}\right)\right)^{q-1}\right) d \mu_{\omega}(x)<\infty,
\end{aligned}
$$

which implies that $\bar{D}_{q}\left(\mu_{\omega}\right)>s_{1}$ almost surely, since the generalized dimensions are determined by the sequence of $r=2^{-k}$.

This method yields similar conclusions for the $L^{q}$-dimensions of the images of measures under other classes of Gaussian process such as fractional Riesz-Bessel motion and infinity scale fractional Brownian motion, see [1]].

\section{$5 \quad$ Measures on almost self-affine sets}

Next we consider $L^{q}$-dimensions of measures on self-affine and almost self-affine sets. For $i=1, \ldots, m$ let $T_{i}$ be linear contractions on $\mathbb{R}^{n}$ and let $\omega_{i}$ be translation vectors. 
The iterated function system $\left\{T_{j}(x)+\omega_{j}\right\}_{j=1}^{m}$ has an non-empty compact attractor $E$ satisfying $E=\cup_{j=1}^{m}\left(T_{j}(E)+\omega_{j}\right)$; such a set is termed a self-affine set. Writing $\boldsymbol{\omega}=\left(\omega_{1}, \ldots, \omega_{m}\right)$ for the set of translations, the attractor $E$ may be characterised in terms of $m$-ary sequences: $E_{\boldsymbol{\omega}}=\bigcup_{\mathbf{j} \in \Lambda^{\infty}} x_{\boldsymbol{\omega}}(\mathbf{j})$, where $x_{\boldsymbol{\omega}}: \Lambda^{\infty} \rightarrow \mathbb{R}^{n}$ is given by the single point in the decreasing intersection

$$
x_{\boldsymbol{\omega}}(\mathbf{j}) \equiv x_{\boldsymbol{\omega}}\left(j_{1}, j_{2}, \ldots\right)=\bigcap_{k=1}^{\infty}\left(T_{j_{1}}+\omega_{j_{1}}\right)\left(T_{j_{2}}+\omega_{j_{2}}\right) \cdots\left(T_{j_{k}}+\omega_{j_{k}}\right)(B),
$$

where $B$ is any ball such that $T_{j}(B)+\omega_{i} \subseteq B$ for all $j$.

Let $p_{1}, \ldots, p_{m}$ be probabilities, so that $0<p_{j}<1$ and $\sum_{j=1}^{m} p_{j}=1$. Let $\mu$ be the Bernoulli probability measure on $\Lambda^{\infty}$ defined by

$$
\mu\left(C_{\mathbf{j}}\right)=p_{j_{1}} p_{j_{2}} \ldots p_{j_{k}} \quad \mathbf{j}=\left(j_{1}, \ldots, j_{k}\right) \in \Lambda^{*}
$$

and extended to a Borel measure on $\Lambda^{\infty}$. For each $\boldsymbol{\omega} \in \Omega$ let $\mu_{\boldsymbol{\omega}}$ be the image measure of $\mu$ under $x_{\boldsymbol{\omega}}$, which is supported by $E_{\boldsymbol{\omega}}$.

We wish to find the generalized dimensions $D_{q}\left(\mu_{\boldsymbol{\omega}}\right)$. This is well-known in the case where the $T_{j}+\omega_{j}$ are similarities and $E_{\boldsymbol{\omega}}$ is a self-similar set. Provided the open set condition is satisfied (that is, there exists a non-empty open set $U$ such that $\cup_{j=1}^{m}\left(T_{j}(U)+\omega_{j}\right) \subset U$ with this union disjoint), then the generalized dimension

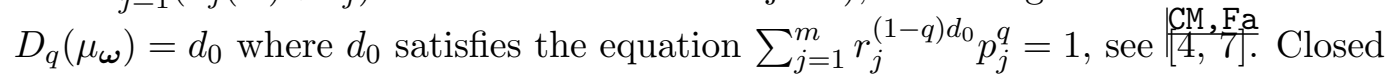
formulae have also been obtained for the generalized dimensions or self-affine 'carpets' and 'sponges', where the $T_{j}$ are all equal and the affine transformations $T_{j_{i}}+\omega_{j_{i}}$ map a given cube onto similarly-aligned rectangles or rectangular parallelepipeds $\left.\frac{1}{16}, 22\right]$.

In general it is difficult to obtain formulae for $L^{q}$-dimensions of measures on self-affine sets, or even for the Hausdorff dimension of the supporting self-affine sets, not least because they need not be continuous in $\boldsymbol{\omega}$. Nevertheless, using a potential-theoretic approach, one may obtain formulae that are valid for almost all $\boldsymbol{\omega}=\left(\omega_{1}, \ldots, \omega_{m}\right)$ in the sense of $m n$-dimensional Lebesgue measure in the case that $1<q \leq 2$, see $[8]$. However, in general there is 'not enough transversality' as $\boldsymbol{\omega}$ varies for the estimates to extend to $q>2$.

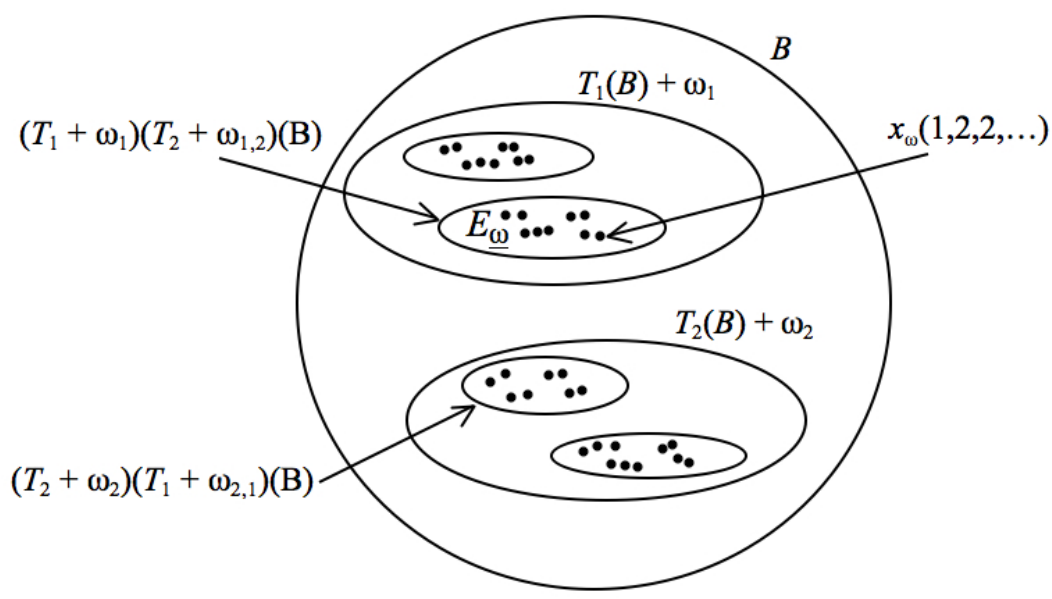

Figure 5: Hierarchical construction of an almost self-affine set $E_{\omega}$ 
One way of circumventing this difficulty is to introduce more randomness by allowing a random perturbation in the translation component at each stage of the construction. We let

$$
\boldsymbol{\omega}=\left\{\omega_{j_{1}, j_{2}, \ldots, j_{k}}:\left(j_{1}, j_{2}, \ldots, j_{k}\right) \in \Lambda^{*}\right\} \in\left(\mathbb{R}^{n}\right)^{\Lambda^{*}}
$$

be a family of translation vectors in $\mathbb{R}^{n}$ which we assume to be bounded. Analogously to $(5.1)$ we let

$$
\begin{aligned}
x_{\boldsymbol{\omega}}(\mathbf{j}) & =\bigcap_{k=1}^{\infty}\left(T_{j_{1}}+\omega_{j_{1}}\right)\left(T_{j_{2}}+\omega_{j_{1}, j_{2}}\right)\left(T_{j_{3}}+\omega_{j_{1}, j_{2}, j_{3}}\right) \cdots\left(T_{j_{k}}+\omega_{j_{1}, j_{2}, \ldots j_{k}}\right)(B(5.4) \\
& =\lim _{k \rightarrow \infty}\left(T_{j_{1}}+\omega_{j_{1}}\right)\left(T_{j_{2}}+\omega_{j_{1}, j_{2}}\right)\left(T_{j_{3}}+\omega_{j_{1}, j_{2}, j_{3}}\right) \cdots\left(T_{j_{k}}+\omega_{j_{1}, j_{2}, \ldots j_{k}}\right)(0) \\
& =\omega_{j_{1}}+T_{j_{1}} \omega_{j_{1}, j_{2}}+T_{j_{1}} T_{j_{2}} \omega_{j_{1}, j_{2}, j_{3}}+\cdots
\end{aligned}
$$

for each $\mathbf{j} \equiv\left(j_{1}, j_{2}, \ldots\right) \in \Lambda^{\infty}$, for some ball $B$ large enough to ensure that $T_{j}(B)+$ $\omega_{j_{1}, j_{2}, \ldots, j_{k}} \subseteq B$ for all $j_{1}, j_{2}, \ldots, j_{k} \in \Lambda^{*}$. We call

$$
E_{\boldsymbol{\omega}}=\bigcup_{\mathbf{j} \in \Lambda^{\infty}} x_{\boldsymbol{\omega}}(\mathbf{j})
$$

an almost self-affine set, see Figure $\frac{\text { almos. }}{5 .}$

$$
\text { almostsa }
$$

We may randomize the translation vectors in the self-affine construction. Assume now that $w_{j_{1}, j_{2}, \ldots, j_{k}}$ in (5.3) are independent identically distributed (i.i.d.) random vectors for $j_{1}, j_{2}, \ldots, j_{k} \in \Lambda^{*}$ with absolutely continuous density with respect to $n$ dimensional Lebesgue measure. We put the product probability measure on $\left(\mathbb{R}^{n}\right)^{\Lambda^{*}}$. We then term $E_{\boldsymbol{\omega}}$ a random almost self-affine set, see Figure 6.

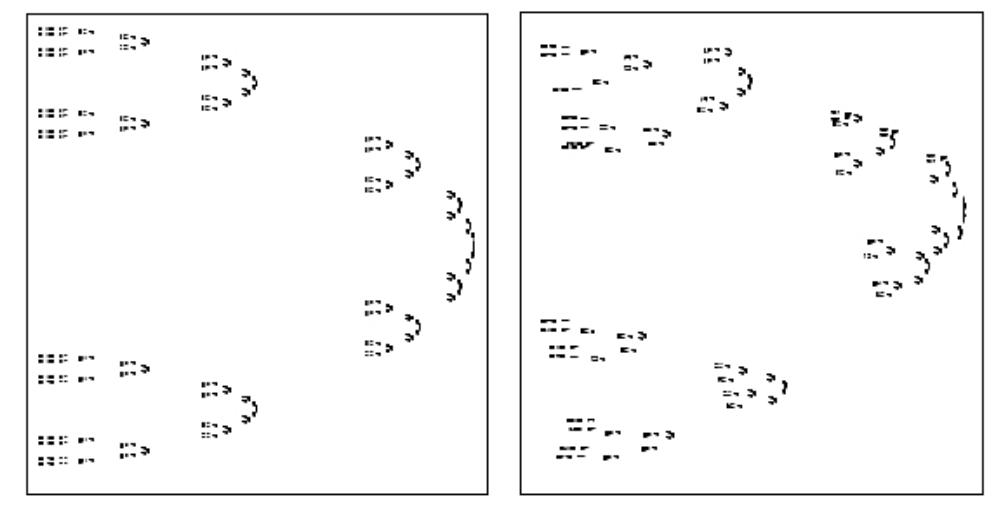

Figure 6: A self-affine set and a random almost self-affine set with the same linear components in the defining transformations

To analyse self-affine and almost self-affine sets we utilize the singular values of the mappings which control the proportions of the components in the construction. The singular values $\alpha_{1} \geq \alpha_{2} \geq \cdots \geq \alpha_{n} \geq 0$ of a linear mapping $T: \mathbb{R}^{n} \rightarrow \mathbb{R}^{n}$ are the positive square roots of the eigenvalues of $T T^{*}$ or equivalently are the semi-axis lengths of the ellipsoid $T(B)$ where $B$ is the unit ball. The singular value function of $T$ is then defined by

$$
\phi^{s}(T)=\alpha_{1} \ldots \alpha_{p-1} \alpha_{p}^{s-p+1}
$$

where $p$ is the integer such that $p-1 \leq s \leq p$. (If $T$ is a similarity then $\phi^{s}(T)$ is just the $s$ th power of the scaling ratio of $T$.) 
There are two important properties of $\phi^{s}$. Firstly it is submultiplicative, that is

$$
\phi^{s}\left(T_{1} T_{2}\right) \leq \phi^{s}\left(T_{1}\right) \phi^{s}\left(T_{2}\right),
$$

and secondly, if $T$ is a fontracting linear map, then $\phi^{s}(T)$ is continuous and strictly decreasing in $s$, see $[5]$. It follows, writing

$$
\Phi_{k}^{s}:=\sum_{i_{1}, \ldots, i_{k} \Lambda^{k}} \phi^{s}\left(T_{i_{1}} \circ \cdots \circ T_{i_{k}}\right)
$$

that $\Phi_{k}^{s}$ itself is also submultiplicative, that is $\Phi_{k+l}^{s} \leq \Phi_{k}^{s} \Phi_{l}^{s}$, so, by the standard property of submultiplicative sequences, the limit

$$
\Phi^{s}:=\lim _{k \rightarrow \infty}\left(\Phi_{k}^{s}\right)^{1 / k}
$$

exists and is decreasing in $s$.

The positive number $d_{0}$ that satisfies $\Phi^{d_{0}}=1$ is called the affinity dimension $d_{0} \equiv d_{0}\left(T_{1}, \cdots, T_{m}\right)$ of the self-affine set $E_{\boldsymbol{\omega}}$ that is the attractor of the IFS of affine maps $\left\{T_{i}+\omega_{i}\right\}_{i=1}^{m}$. In other words $d_{0}$ is given by

$$
\Phi^{d_{0}}\left(T_{1}, \ldots, T_{m}\right) \equiv \Phi^{d_{0}}=\lim _{k \rightarrow \infty}\left(\sum_{i_{1} \ldots i_{k} \in \Lambda^{k}} \phi^{d_{0}}\left(T_{i_{1}} \circ \cdots \circ T_{i_{k}}\right)\right)^{1 / k}=1 ;
$$

notice that the affinity dimension depends only on the linear parts of the IFS functions. Affinity dimensions provide 'generic' values for the Hausdorff and boxcounting dimensions of self-affine sets. We write $\operatorname{dim}_{\mathrm{H}}$ and $\overline{\operatorname{dim}}_{\mathrm{B}}$ for Hausdorff and upper box-counting dimensions respectively

Proposition 5.1 Let $E_{\boldsymbol{\omega}}$ be a self-affine or almost self-affine subset of $\mathbb{R}^{n}$. Then

$$
\operatorname{dim}_{\mathrm{H}} E_{\boldsymbol{\omega}} \leq \overline{\operatorname{dim}}_{\mathrm{B}} E_{\boldsymbol{\omega}} \leq d_{0}\left(T_{1}, \cdots, T_{m}\right)
$$

where $\left(T_{1}, \cdots, T_{m}\right)$ are the linear parts of the affine contractions in the construction of $E_{\boldsymbol{\omega}}$. If $E_{\boldsymbol{\omega}}$ is self-affine with $\left\|T_{j}\right\|<\frac{1}{2}$ for all $j$ then there is equality in (5.8) for almost all translation sets $\boldsymbol{\omega}_{\boldsymbol{\omega}} \in\left(\mathbb{R}^{n}\right)^{m}$. If $E_{\boldsymbol{\omega}}$ is a random almost self-affine set then there is equality in (5.8) for almost all $\boldsymbol{\omega} \in \Omega$ with no restriction on $\left\|T_{j}\right\|$.

Proof. Inequality ( $\frac{\text { dimineq }}{5.8)}$ is obtained by a covering method. Almost sure equality for self-affine sets and random almost self-affine sets may J $_{\text {Fas }}$ derived from energy estimates for measures supported on the sets, see $\left[\frac{5]}{5]}\right.$ and $\left.\frac{12}{12}\right]$ for the two settings.

To obtain generic formulae for $L^{q}$-dimensions, we adapt the definition of affine dimension. With $\mu$ to be a Bernoulli measure on $\Lambda^{\infty}$ defined by $\left(\frac{m}{5.2}\right)$, let

$$
\begin{aligned}
\Phi_{q}^{s} & =\lim _{k \rightarrow \infty}\left(\sum_{i_{1}, \ldots, i_{k} \in \Lambda^{k}} \phi^{s}\left(T_{i_{1}} \circ T_{i_{2}} \circ \cdots \circ T_{i_{k}}\right)^{1-q} \mu\left(C_{i_{1}, i_{2}, \ldots, i_{k}}\right)^{q}\right)^{1 / k} \\
& =\lim _{k \rightarrow \infty}\left(\sum_{i_{1}, \ldots, i_{k} \in \Lambda^{k}} \phi^{s}\left(T_{i_{1}} \circ T_{i_{2}} \circ \cdots \circ T_{i_{k}}\right)^{1-q}\left(p_{i_{1}} p_{i_{2}} \ldots p_{i_{k}}\right)^{q}\right)^{1 / k} .
\end{aligned}
$$

Again the limits exist as a consequence of supermultiplicativity, and if $q>1$ then $\Phi_{q}^{s}$ is strictly increasing and continuous in $s$. Thus we may define positive numbers $d_{q}$ by the requirement that

$$
\Phi_{q}^{d_{q}}=1
$$


As before we write $\mu_{\boldsymbol{\omega}}$ for the image of the Bernoulli measure $\mu$ under $x_{\boldsymbol{\omega}}$. We refer to $\mu_{\boldsymbol{\omega}}$ as a self-affine measure for $\boldsymbol{\omega} \in \Lambda$ when the support $E_{\boldsymbol{\omega}}$ is a self-affine set, and as an (random) almost self-affine measure when $\boldsymbol{\omega} \in \Omega$ and the support is a (random) almost self-affine set.

prop1q2 Proposition 5.2 Let $1<q \leq 2$. Let $\mu$ be a Bernoulli measure on $\Lambda^{\infty}$. For every self-affine or almost self-affine measure $\mu_{\boldsymbol{\omega}}$ on $\mathbb{R}^{n}$

$$
\bar{D}_{q}\left(\mu_{\boldsymbol{\omega}}\right) \leq \min \left\{d_{q}, n\right\}
$$

where $d_{q}$ is given by $\frac{\text { dqdef }}{(5.10) .}$ Moreover, $D_{q}\left(\mu_{\omega}\right)$ exists and

$$
D_{q}\left(\mu_{\boldsymbol{\omega}}\right)=\min \left\{d_{q}, n\right\}
$$

in the self-affine case provided $\left\|T_{j}\right\|<\frac{1}{2}$ for all $j$, for almost all $\boldsymbol{\omega} \in\left(\mathbb{R}^{n}\right)^{m}$, and also in the random almost self-affine case for almost all $\boldsymbol{\omega} \in\left(\mathbb{R}^{n}\right)^{\Lambda^{*}}$ (with no restriction on the $\left.\left\|T_{j}\right\|\right)$.

Note on proof. This is proved in $\left[\begin{array}{l}F_{2} 5 \\ 8\end{array}\right.$ using a potential-theoretic method; the proof adapts easily to give equality in the random almost self-affine case.

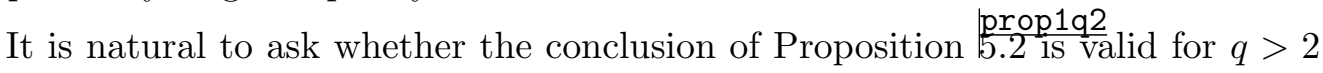
when the basic potential-theoretic method is inadequate. This higher moment case can be addressed using the inequality of Section 3 . However, for self-affine measures $\mu_{\omega}$ there is not-enough randomness or transversality to get an adequate estimate in (3.1) to lead to equality for almost all $\boldsymbol{\omega} \in\left(\mathbb{R}^{n}\right)^{m}$. Thus we can only obtain the lower bound for random almost self-affine measures.

Theorem 5.3 Let $q>1$. Let $\mu$ be a Bernoulli measure on $\Lambda^{\infty}$. For every self-affine or almost self-affine measure $\mu_{\boldsymbol{\omega}}$ on $\mathbb{R}^{n}$

$$
\bar{D}_{q}\left(\mu_{\boldsymbol{\omega}}\right) \leq \min \left\{d_{q}, n\right\}
$$

where $d_{q}$ is given by (15.10). If $\mu_{\boldsymbol{\omega}}$ is a random almost self-affine measure then $D_{q}\left(\mu_{\boldsymbol{\omega}}\right)$ exists and

$$
D_{q}\left(\mu_{\boldsymbol{\omega}}\right)=\min \left\{d_{q}, n\right\}
$$

for almost all $\boldsymbol{\omega} \in\left(\mathbb{R}^{n}\right)^{\Lambda^{*}}$.

Sketch of proof. The upper bound $\left(\begin{array}{l}\text { dqeq1 } \\ 512\end{array}\right) \operatorname{comes}$ from splitting ellipses of the form that occur in the intersections in (5.4) into appropriate pieces and summing the powers of the measures, see $[8,9]$.

For the case where $q \geq 2$ is an integer and $\mu_{\boldsymbol{\omega}}$ a random almost self-affine set, let $\mathbf{j}_{1}, \ldots, \mathbf{j}_{q} \in \Lambda^{\infty}$. Using the geometry and randomness or higher transversality available in the construction, we may obtain an estimate

$$
\mathrm{P}\left\{\left|x_{\omega}\left(\mathbf{j}_{q}\right)-x_{\omega}\left(\mathbf{j}_{j}\right)\right| \leq r \text { for all } j\right\} \leq c r^{s(q-1)} \phi^{s}\left(T_{\mathbf{i}_{1}}\right)^{-1} \phi^{s}\left(T_{\mathbf{i}_{2}}\right)^{-1} \ldots \phi^{s}\left(T_{\mathbf{i}_{q-1}}\right)^{-1}
$$

where $\mathbf{i}_{1}, \ldots, \mathbf{i}_{q-1}$ are the join points of $\mathbf{j}_{1}, \ldots, \mathbf{j}_{q}$. Using $(2.5)$ we get, for all $s>0$,

$$
\begin{aligned}
& \mathrm{E} \int \mu_{\omega}(B(x, r))^{q-1} d \mu_{\omega}(x) \\
& \quad \leq c r^{s(q-1)} \int \cdots \int \phi^{s}\left(T_{\mathbf{i}_{1}}\right)^{-1} \phi^{s}\left(T_{\mathbf{i}_{2}}\right)^{-1} \ldots \phi^{s}\left(T_{\mathbf{i}_{q-1}}\right)^{-1} d \mu\left(\mathbf{j}_{1}\right) \ldots d \mu\left(\mathbf{j}_{q}\right) \\
& \quad \leq c r^{s(q-1)}\left(\sum_{k=0}^{\infty} p(k)\left[\sum_{|\mathbf{i}|=k} \phi^{s}\left(T_{\mathbf{i}}\right)^{1-q} \mu\left(C_{\mathbf{i}}\right)^{q}\right]^{\frac{1}{q-1}}\right)^{q-1}
\end{aligned}
$$


for some polynomial $p$, taking $f(\mathbf{i})=\phi^{s}\left(T_{\mathbf{i}}\right)^{-1}$ in inequality $\left(\begin{array}{l}\text { ineq } \\ 3.3)\end{array}\right.$ tion $(5.9),(5.10)$ of $\Phi_{q}^{s}$, this series converges if $0<s<d_{q}$, in which case

$$
\begin{aligned}
\mathrm{E} \sum_{k=1}^{\infty} 2^{-s_{1}(q-1)} \int \mu_{\omega}\left(B\left(x, 2^{-k}\right)\right)^{q-1} d \mu_{\omega}(x) \\
=\mathrm{E} \int\left(\sum_{k=1}^{\infty} 2^{-s_{1}(q-1)} \mu_{\omega}\left(B\left(x, 2^{-k}\right)\right)^{q-1}\right) d \mu_{\omega}(x)<\infty,
\end{aligned}
$$

for all $0<s_{1}<s$, giving $D_{q}\left(\mu_{\boldsymbol{\omega}}\right)>s_{1}$ for all $s_{1}<d_{q}$, as required.

For full details of this argument and the case of non-integer $q>1$ see $\left[\begin{array}{l}\text { Fa7 } \\ {[10]}\end{array}\right.$.

\section{Random multiplicative cascade measures}

Let $\Lambda=\{1,2, \ldots, m\}$, let $W_{\mathbf{i}}$ be independent positive random variables indexed by $\mathbf{i}=i_{1}, i_{2}, \ldots, i_{k} \in \Lambda^{*}$ and let

$$
X_{\mathbf{i}}=W_{i_{1}} W_{i_{1}, i_{2}} \cdots W_{i_{1}, i_{2}, \ldots, i_{k}} .
$$

We may identify the cylinders in symbolic space with the hierarchy of $m$-ary subintervals of of $[0,1]$ in the obvious way, see Figure 6 . We assume that $\mathrm{E}\left(W_{\mathbf{i}}\right)=1$ for all

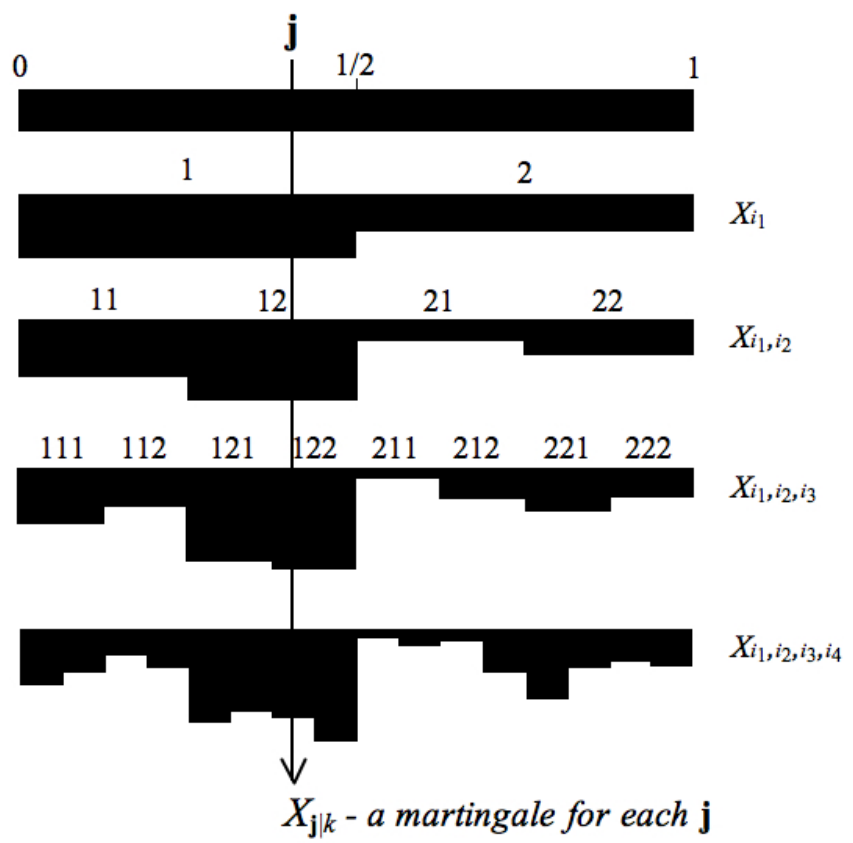

Figure 7: A random multiplicative binary cascade measure represented on the interval $[0,1]$, with $X_{\mathbf{j} \mid k}$ a martingale for each $\mathbf{j} \in[0,1]$.

$\mathbf{i} \in \Lambda^{*}$ in which case $\left(X_{\mathbf{j} \mid k}, \mathcal{F}_{k}\right)$ is a martingale for each $\mathbf{j} \in \Lambda^{\infty}$, where $\mathbf{j} \mid k$ denotes the curtailment of $\mathbf{j}$ after $k$ terms and $\mathcal{F}_{k}$ is the $\sigma$-field generated by $\left\{W_{\mathbf{i}}: \mathbf{i} \in \cup_{l=1}^{k} \Lambda^{l}\right\}$.

These martingales, termed random multiplicative cascade measures, were introduced and studied in the 1970s by Mandelbrot $[19]$ and Kahane and Peyrière $[13,14]$ 
who obtained many properties in the 'self-similar' case, that is when the $W_{\mathbf{i}}$ are independent and identically distributed. Let $\mu$ be a Borel probability measure on $\Lambda^{\infty}$. Of particular interest are $k$-th level sums

$$
\sum_{|\mathbf{i}|=k} X_{\mathbf{i}} \mu\left(C_{\mathbf{i}}\right) \equiv \int X_{\mathbf{j} \mid k} d \mu(\mathbf{j}),
$$

which moments of the sums remain bounded as $k \rightarrow \infty$ and in what setting the integral converges. It follows from Minkowski's inequality that if $\mathrm{E}\left(\left(\sum_{\mathbf{i} \in \Lambda^{k}} X_{\mathbf{i}} \mu\left(C_{\mathbf{i}}\right)\right)^{q}\right)$ is bounded in $k$ then so is $\sum_{\mathbf{i} \in \Lambda^{k}} \mathrm{E}\left(\left(X_{\mathbf{i}} \mu\left(C_{\mathbf{i}}\right)\right)^{q}\right)$ ma so of more interest are opposite implications. Using the inequality from Section 3 we get the following result.

Theorem 6.1 Let $q>1$ be a real number. If

$$
\limsup _{k \rightarrow \infty}\left(\sum_{|\mathbf{i}|=k} \mathrm{E}\left(X_{\mathbf{i}}^{q}\right) \mu\left(C_{\mathbf{i}}\right)^{q}\right)^{1 / k}<1
$$

then

$$
\limsup _{k \rightarrow \infty} \mathrm{E}\left(\left(\sum_{|\mathbf{i}|=k} X_{\mathbf{i}} \mu\left(C_{\mathbf{i}}\right)\right)^{q}\right)<\infty
$$

and $\int X_{\mathbf{j} \mid k} d \mu(\mathbf{j})$ converges a.s. and in $L^{q}$. Note that we require the underlying $W_{\mathbf{i}}$ to be independent but not necessarily identically distributed.

This, and, ather properties of these martingales were obtained by Kahane and Peyrière $[13,14]$ when the random cascade is 'self-similar', that is when the $W_{\mathbf{i}}$ are identically distributed, utilizing the self-similarity to show that the sums satisfy a random difference equation. There have been many subsequent extensions and variants, see [1, 18] which contain many further references. Barral [1] proved this result without the i.i.d. requirement on the $W_{\mathbf{i}}$ in the case $1<q \leq 2$, with the martingales defined in a more general continuous, rather than discrete, setting.

Note on the proof of Theorem $\frac{\text { randcas }}{6.1}$. When $q>1$ is an integer we may expand

$$
\begin{aligned}
\mathrm{E}\left(\left(\sum_{|\mathbf{i}|=k} X_{\mathbf{i}} \mu\left(C_{\mathbf{i}}\right)\right)^{q}\right) & =\sum_{\substack{\left|\mathbf{i}_{1}\right|,\left|\mathbf{i}_{2}\right|, \ldots,\left|\mathbf{i}_{q}\right|=k\\
}} \mathrm{E}\left(X_{\mathbf{i}_{1}} X_{\mathbf{i}_{2}} \cdots X_{\mathbf{i}_{q}}\right) \mu\left(C_{\mathbf{i}_{1}}\right) \mu\left(C_{\mathbf{i}_{2}}\right) \cdots \mu\left(C_{\mathbf{i}_{q}}\right) \\
& \leq\left(\sum_{k=0}^{\infty} p(k)\left(\sum_{|\mathbf{i}|=k} \mathrm{E}\left(X_{\mathbf{i}}^{q}\right) \mu\left(C_{\mathbf{i}}\right)^{q}\right)^{\frac{1}{q-1}}\right)^{q-1} .
\end{aligned}
$$

for a polynomial $p$, where this inequality may be established using induction in a manner akin to that of Theorem 3.1 by relating the expectations of products of the $X_{\mathbf{i}_{j}}$ to expectations of powers of the $X_{\mathbf{i}}$ at the join points of $\mathbf{i}_{1}, \ldots, \mathbf{i}_{q}$. The conclusion $(\overline{6.2})$ then follows from ( $\overline{6.1)}$.

As with Theorem 3.1 the argument for non-integer $q$ requires a more involved induction argument.

\section{References}

BM [1] J. Barral and B.B. Mandelbrot. Random multiplicative multifractal measures I, II, III, in Fractal Geometry and Applications: A Jubilee of Benoît Mandelbrot (M. Lapidus and M. V. Frankenkuijsen, eds.), Proc. Sympos. Pure Math. 72 (2004) 3-90. 
Man

[2] R.J. Adler. The Geometry of Random Fields, (John Wiley, 1981).

[3] S.M. Berman. Local nondeterminism and local times of Gaussian processes, Indiana Univ. Math. J. 23 (1973), 69-94.

[4] R. Cawley and R.D. Mauldin. Multifractal decomposition of Moran fractals, Adv. Math. 92 (1992), 196-236.

[5] K.J. Falconer. The Hausdorff dimension of self-affine fractals, Math. Proc. Cambridge Philos. Soc. 103 (1988), 339-350.

[6] K.J. Falconer. Techniques in Fractal Geometry, (John Wiley, 1997).

[7] K.J. Falconer. Fractal Geometry-Mathematical Foundations and Applications, 2nd Ed., (John Wiley, 2003).

[8] K.J. Falconer. Generalized dimensions of measures on self-affine sets, Nonlinearity 12 (1999), 877-891.

[9] K.J. Falconer. Generalised dimensions of measures on almost self-affine sets, Nonlinearity 23 (2010), 1047-1069.

[10] K.J. Falconer. Higher moments of random multiplicative cascade measures, in preparation.

[11] K.J. Falconer and Y.Xiao. Generalized dimensions of images of measures under Gaussian processes, arXiv:1212.2383.

[12] Jordan, T., Pollicott, M., Simon, K.: Hausdorff dimension for randomly perturbed self affine attractors, Commun. Math. Phys. 270 (2007), 519-544.

[13] J.P. Kahane. Sur le chaos multiplicatif, Ann. Sci. Math. Québec 9 (1985) 105150.

[14] J.P. Kahane and J. Peyrière. Sur certaines martingales de Benoit Mandelbrot, Adv. Math. 22 (1976), 131-145.

[15] J.-P. Kahane. Some Random Series of Functions, 2nd Ed., (Cambridge University Press, 1985).

[16] J. King. The singularity spectrum for general Sierpinski carpets, Adv. Math. 116 (1995), 1-8.

[17] K.-S. Lau. Self-similarity, $L^{p}$-spectrum and multifractal formalism, in: Fractal Geometry and Stochastics (Eds. C. Bandt, S. Graf and M. Zähle, Progress in Probability 37, pp. 55-90 (Birkhäuser, 1995).

[18] Quansheng Liu. On generalized multiplicative cascades, Stoch. Processes Appl. 86 (2000), 263-286.

[19] B. Mandelbrot. Intermittent turbulence in self-similar cascades: Divergence of higher moments and dimension of the carrier, J. Fluid. Mech. 62 (1974), 331-358.

[20] B.B. Mandelbrot and J.W. Van Ness. Fractional Brownian motions, fractional noises and applications, SIAM Review 10 (1968), 422-437.

[21] P. Mattila. Geometry of Sets and Measures in Euclidean Spaces, (Cambridge University Press, 1999).

[22] L. Olsen. Self-affine multifractal Sierpinski sponges in $\mathbb{R}^{d}$, Pacific J. Math. 183 (1998), 143-199. 
[23] Y. Peres and B. Solomyak. Existence of $L^{q}$ dimensions and entropy dimension for self-conformal measures, Indiana Univ. Math. J. 49 (2000), 1603-1621.

PSc [24] Y. Peres and B. Schlag. Smoothness of projections, Bernoulli convolutions, and the dimension of exceptions, Duke Math. J. 102 (2000), 193-251.

Xi06 [25] Y. Xiao. Properties of local nondeterminism of Gaussian and stable random fields and their applications, Ann. Fac. Sci. Toulouse Math. XV (2006), 157193.

[26] Y. Xiao. Properties of strong local nondeterminism and local times of stable random fields, in: Seminar on Stochastic Analysis, Random Fields and Applications VI, (Eds: R.C. Dalang, M. Dozzi and F. Russo), Progress in Probability 63, pp. 279-310 ( Birkhäuser, Basel, 2011). 